Fukushima J. Med. Sci.,

Vol. 66, No. 2, 2020

[Original Article]

\title{
Exhaled carbon monoxide levels in infants and toddlers with episodic asthma
}

\author{
Yoichiro Ohara $^{1)}$, Takahiro Ohara ${ }^{2)}$, Koichi Hashimoto ${ }^{3)}$ and Mitsuaki Hosoya ${ }^{3)}$ \\ ${ }^{1)}$ Ohara Children's Clinic, ${ }^{2)}$ Division of Community Medicine, Tohoku Medical and Pharmaceutical \\ University, ${ }^{3)}$ Department of Pediatrics, Fukushima Medical University
}

(Received January 15, 2019, accepted May 20, 2020)

\begin{abstract}
Objective : There are few lung function tests available to evaluate bronchial asthma in infants and toddlers. The objective of this study was to test the hypothesis that the measurement of exhaled carbon monoxide (eCO) levels is applicable to evaluate infants and toddlers with stable asthma and during acute asthma attack.

Methods : A one-way valve breath sampling bag was developed to collect the exhaled air of infants and toddlers. A total of 483 infants (under 2 years) and toddlers (2-5 years) were studied ; 355 had an established diagnosis of asthma (182 suffering mild asthma attacks and 173 without active asthmatic symptoms), 119 had upper respiratory infection (URI) including acute bronchitis, and 9 were healthy.

Results : In infants and toddlers, eCO levels of those with asthma attacks [median (interquartile range $)=2.0(2.0-3.25) \mathrm{ppm}, n=182]$ were significantly higher than those of subjects with asymptomatic asthma [2.0 (1.0-2.0) ppm, $n=173, P<0.0001$ ], URI [2.0 (1.0-3.0) ppm, $n=119, P<0.0001]$, and healthy children $[1.0(0.0-1.0) \mathrm{ppm}, n=9, P<0.0001]$. In 75 children with asthma petit mal, eCO levels during asthma attacks $[3.0(2.0-4.0) \mathrm{ppm}]$ significantly decreased after therapy [1.0 (1.0$2.0) \mathrm{ppm}, P<0.0001]$. In infants and toddlers with an established diagnosis of asthma $(n=355)$, eCO cut-off $>2$ ppm discriminated asthma attack from an asymptomatic state with a sensitivity of $95.6 \%$, a specificity of $43.3 \%$, and an area under the curve (AUC) of 0.71 (95\% CI : $0.65-0.76, P<$ 0.0001). In 401 infants and toddlers with some respiratory symptoms, of which 285 cases were finally diagnosed as asthma [eCO level $=2.0(2.0-3.0) \mathrm{ppm}]$ and 116 cases were not asthma [eCO level $=2.0(1.0-3.0) \mathrm{ppm}, P<0.0001]$, eCO cut-off $>3 \mathrm{ppm}$ supported the final diagnosis of asthma with a sensitivity of $38.9 \%$, a specificity of $74.1 \%$, and AUC of 0.63 (95\% CI : $0.56-0.69, P<0.0001$ ). Conclusion : The measurement of eCO by a novel method is applicable to evaluate asthmatic activity and treatment responsiveness, and to diagnose asthma in infants and toddlers.
\end{abstract}

Key words : infants and toddlers, mild asthma attack, simple breath sampling method

\section{Introduction}

Asthma is a chronic lung disorder characterized by airway inflammation ${ }^{1)}$. The inducible form of heme oxygenase (HO)- 1 is expressed in airway epithelial cells ${ }^{2)}$, endothelial cells ${ }^{3)}$, and alveolar macrophages $^{4)}$. Carbon monoxide (CO) is produced en- dogenously by HO-1, and is known to be present in a low concentration in the exhaled air of normal subjects $^{5,6)}$.

Upregulation of HO-1 by oxidative stress and proinflammatory cytokines in the airways and lung parenchyma increases the levels of exhaled CO $(\mathrm{eCO})$ in patients with inflammatory pulmonary dis-

Corresponding author: Yoichiro Ohara E-mail : znthstay@zd5.so-net.ne.jp

(C2020 The Fukushima Society of Medical Science. This article is licensed under a Creative Commons [Attribution-NonCommercial-ShareAlike 4.0 International] license.

https://creativecommons.org/licenses/by-nc-sa/4.0/ 
eases such as bronchiectasis, upper respiratory tract infection (URI), acute pneumonia, silicosis, and seasonal allergic rhinitis ${ }^{7-13)}$. Recently, eCO levels were shown to be elevated in adults ${ }^{4,6)}$, and school-age, and preschool children ${ }^{14-16)}$ with persistent asthma.

Our group has investigated the usefulness of eCO measurement in school- and preschool children $^{15,16)}$. In infants and toddlers, however, measuring the eCO concentrations of episodic asthma in acute asthma attacks or in stable conditions and for other respiratory diseases is not feasible using the conventional method ${ }^{17)}$. We developed a simple sampling system, which allows the measurement of eCO concentration in infants and toddlers with asthma with or without attacks or those with URI. We examined the hypothesis that eCO reflects disease status and treatment effects in infants and toddlers.

\section{Methods}

\section{Study design and subjects}

Infants (aged under 2 years) and toddlers (aged 2-5 years) with asthma, with or without attacks, those with URI including acute bronchitis, and healthy controls were recruited at Ohara Children's Clinic, Fukushima, from June 2013 to December 2016. We enrolled only infants and toddlers with mild asthma attack as classified by the Japanese Guideline for the Diagnosis and Treatment of Allergic Diseases 2010 (JAGL 2010) $^{18)}$.

\section{Definition and diagnosis of asthma}

Infantile asthma is defined as asthma affecting infants aged $<2$ years. A diagnosis of infantile asthma is clinically made if there are 3 or more episodes of marked expiratory wheezing, regardless of the presence of respiratory tract infection, considering family history, past history, serum IgE level, response to $\beta 2$ stimulant inhalation, and so forth ${ }^{18)}$. Intermittent asthma is defined as occurrence of symptoms fewer than several times a year and only brief exacerbations ${ }^{18)}$ in infants and toddlers with mild asthma attack. We measured serum IgE levels in asthmatic subjects using a commercially available method. We followed these children to control their symptoms ${ }^{15)}$. The criteria of mild asthma attack included breathless wheezing $(+/-)$, retraction $(-)-(+)$, prolongation of expiration $(-)$, supine posture $(-)$, cyanosis $(-)$ and $\mathrm{SpO}_{2} \geq 96 \%$. We followed these infants and toddlers to control their symptoms. We defined asymptomatic asthma as a history of infrequent mild asthma without exacerba- tions for at least 8 weeks before the study ${ }^{15,16)}$. No asthma patients had any respiratory history other than asthma. We excluded children from the study whose parents were current smokers to avoid the influence of second-hand smoke on eCO concentration.

This study was conducted in accordance with the Declaration of Helsinki, and was approved by the ethics committee of Fukushima Medical University (Number 1432) on May 28, 2012. Informed parental consent was documented in writing.

Collection of exhaled air and eCO measurement in infants and toddlers

We used a breath-sampling bag $(200 \mathrm{~mL})$ with a one-way valve, which is usually used for the $13-\mathrm{C}$ urea exhaled test (Otsuka Pharmaceutical, Tokyo, Japan ${ }^{15)}$. We cut the bottom two thirds of the bag and connected it with a polyvinyl bag $(18 \mathrm{~cm} \times 27$ $\mathrm{cm} \times 2 \mathrm{~cm}$, approximately $1 \mathrm{~L}$ in volume) to decrease the respiratory resistance (Fig. 1).

After collecting exhaled air, the sampling bag was connected to a piCO+Smokerlyzer (Bedfont Scientific Ltd. England) using the one-way valve release adaptor and manually expelled the air into the instrument to measure eCO concentration (Fig. 2). The measurement range of the piCO+Smokerlyzer is 0-150 ppm. The instrument was calibrated with a gaseous mixture containing $20 \mathrm{ppm} \mathrm{CO}$ according to the manufacturer's instructions. We confirmed the background $\mathrm{CO}$ level in the clinic using a Fluke CO220 Carbon Monoxide Meter (Everett, WA, USA) to be $0 \mathrm{ppm}$. We measured eCO only once, because it was impossible to measure eCO more than once with infants and toddlers.

\section{Clinical managements of the patients}

Patients were treated with combined inhalation therapy with salbutamol and sodium cromoglicate (SCG) according to guidelines for the treatment of mild asthma attack. The combination therapy consisted of a mixture of either $0.1 \mathrm{~mL}$ ( $<1$ year of age), $0.2 \mathrm{~mL}$ ( $1-5$ years of age) of $5 \mathrm{mg} / \mathrm{mL}$ aqueous solution of salbutamol and $2 \mathrm{~mL}$ of $1 \%$ aqueous solution of SCG, which was delivered for 3 min using a jet nebulizer ${ }^{19,20)}$. SCG + salbutamol inhalation therapy was repeated a maximum of four times per day if symptoms persisted. Long-term asthma management with anti-inflammatory drugs was performed following the JPGL 2012 guideline ${ }^{18)}$. We also measured arterial oxygen saturation using pulse oxymetry $\left(\mathrm{SpO}_{2}\right)$ with a pulse oxymeter ${ }^{11)}$ simultaneously with measurement of eCO concentration to monitor 


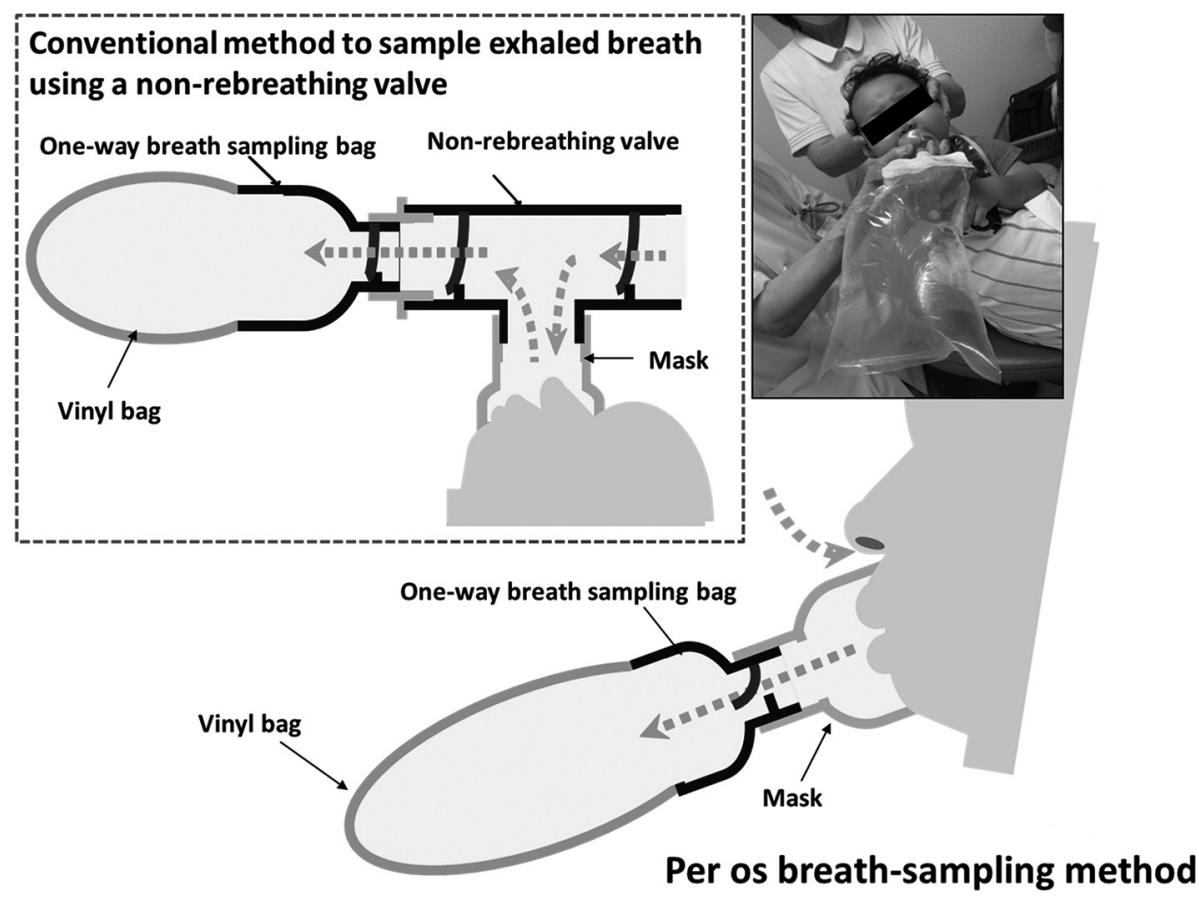

Figure 1. Breath sampling methods

Left-upper square enclosed with a dotted line : conventional method to sample exhaled breath using a non-rebreathing valve.

Right-upper square enclosed with a line and lower figure : per os breath sampling method.
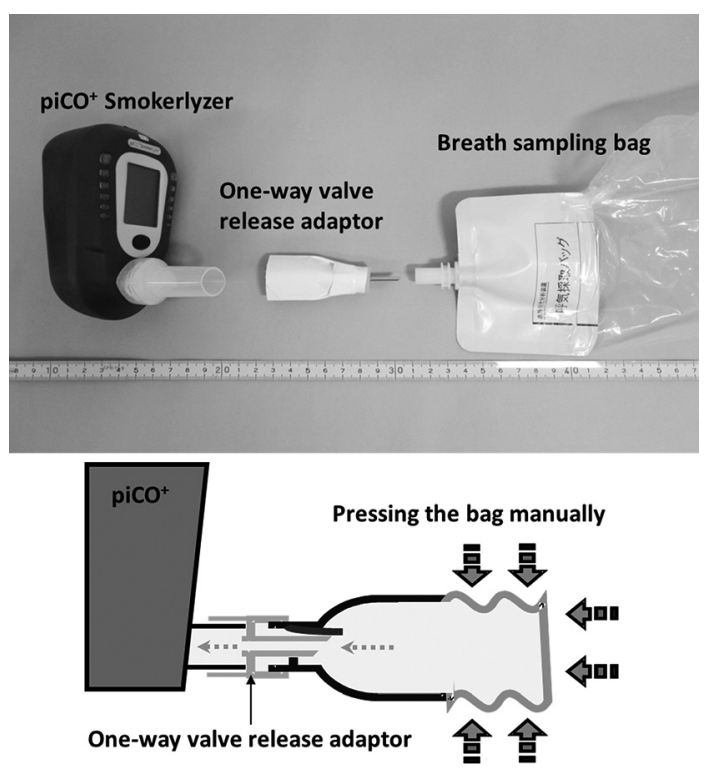

Figure 2. eCO measurement of collected exhaled air After collecting exhaled air, the sampling bag was connected to the Pico Smokerlyzer using a oneway valve release adaptor and manually pushed out inspired air into the instrument to measure eCO concentration.

eCO, exhaled carbon monoxide.

respiratory conditions in patients with an acute asthma attack. We excluded subjects from the study whose respiratory conditions prevented complete filling of the sampling bags.

\section{Statistical analysis}

Numerical data are presented as median (interquartile range). Numerical data were compared among groups using the Kruskal-Wallis test with Dunn's multiple comparisons test or the Mann Whitney test, as appropriate. Categorical data were compared among groups using a chi-squared test or Fisher's exact test, as appropriate. Because the levels of eCO did not show the normal distribution assessed by the D'Agostino \& Pearson omnibus normality test, eCO data were compared among groups using the Kruskal-Wallis test with Dunn's multiple comparisons test or the Mann Whitney test, as appropriate. Changes between eCO during asthma attacks and after treatment were analyzed using the Wilcoxon matched-pairs signed rank test. A value of $P<0.05$ was considered significant.

We analyzed eCO levels in infants and toddlers in 4 ways : 1) comparison of eCO levels among healthy infants and toddlers, those with URI, those diagnosed as asthma but asymptomatic at the time of the eCO measurement (asymptomatic asthma group), and those diagnosed as asthma and in mild attack at the time of the $\mathrm{eCO}$ measurement (asthma attack group) ; 2) comparison of eCO levels before and after the treatment in 75 infants and toddlers 
with asthma attack in whom both the eCO values were available ; 3 ) to test the ability of eCO to diagnose activity of asthma, we compared eCO values in the asymptomatic asthma group and the asthma attack group and performed receiver operating characteristics (ROC) analyses ; 4) to test the ability of $\mathrm{eCO}$ to detect infants and toddlers with a final diagnosis of asthma in those with any respiratory symptoms, we compared $\mathrm{eCO}$ values in those with any respiratory symptoms with a final diagnosis of asthma (composed from the asthma attack group and part of the asymptomatic asthma group with any respiratory symptoms not satisfying the criteria of asthma attack) and those with respiratory symptoms without the final diagnosis of asthma (URI group, excepting 3 cases without respiratory symptoms), and performed ROC analyses.

$\mathrm{ROC}$ analyses of eCO to discriminate disease activity and the final diagnosis of asthma were performed to obtain the area under the curve (AUC). Cut-off values were determined to maximize the sum of the sensitivity and specificity. To assess independence of eCO to discriminate disease activity and the final diagnosis of asthma, multiple logistic regression analyses were performed with adjustments by age in years, gender, and $\mathrm{SpO}_{2}$ values.

\section{Results}

\section{Patient background and the eCO levels in asthma and other status}

We enrolled a total of 355 infants and toddlers with asthma, in whom 182 had mild asthma attacks and 173 were asymptomatic. We also enrolled 119 infants and toddlers with URI - including acute bron- chitis - and 9 healthy infants. Sampling and eCO measurement were accomplished in all subjects.

Healthy infants and toddlers were younger than those in other groups (Table 1). Children in the asthmatic group were older than those in the URI group. As such, body weights in the asthmatic group were generally higher than in other groups. $\mathrm{SpO}_{2}$ of the asthma attack group was lower than other groups, however it was within the normal range, which indicated that the respiratory state of the present patients was not severely depressed. The infants and toddlers with asthma had been taking a disodium cromoglycate inhalation in $\approx 60 \%$, and a leukotriene receptor antagonist in $\approx 90 \%$, of both the asymptomatic asthma and asthma attack groups. The infants and toddlers in the asthma attack group had been taking an inhaled corticosteroid more often $(10 \%)$ than those in the asymptomatic asthma group $(3 \%, P=0.0005)$.

The eCO level during asthma attacks in asthmatic infants and toddlers $[n=182$, median (interquartile range $)=2.0(2.0-3.25) \mathrm{ppm}]$ was significantly higher compared to healthy controls $[n=9$, $1.0(0.0-1.0)$ ppm, $P<0.0001]$, URI $[n=119,2.0$ (1.0-3.0) ppm, $P<0.0001]$ and those with asymptomatic asthma $[n=173,2.0(1.0-2.0)$ ppm, $P<$ 0.0001] (Fig. 3). However, there were considerable overlaps in the eCO levels among the asthma attack, asymptomatic asthma, and URI groups (Fig. $3)$. The infants and toddlers taking an inhaled corticosteroid tended to have a higher eCO level [2.0 $(1.0-4.75) \mathrm{ppm}]$ than those not taking [2.0 (2.0-3.0), $P=0.53]$. Neither taking a disodium cromoglycate inhalation nor a leukotriene receptor antagonist related with eCO levels. There were no gender differences in the eCO levels within the subgroups

Table 1. Subjects and characteristics

\begin{tabular}{|c|c|c|c|c|c|}
\hline & $\begin{array}{l}\text { Healthy } \\
n=9\end{array}$ & $\begin{array}{c}\text { URI } \\
n=119\end{array}$ & $\begin{array}{c}\text { Asymptomatic } \\
\text { Asthma } \\
n=173\end{array}$ & $\begin{array}{c}\text { Asthmatic Attack } \\
n=182\end{array}$ & $P$ \\
\hline Age (years)* & $0.85(0.78-0.86)$ & $1.0(0.7-1.9)$ & $2.8(2.2-3.6)$ & $2.5(2.0-3.4)$ & $<0.0001$ \\
\hline Female, $n(\%)$ & $3(33)$ & $43(36)$ & $40(30)$ & $61(34)$ & 0.0704 \\
\hline Body weight $(\mathrm{kg}) *$ & $9.1(8.5-9.3)$ & $9.0(8.4-10.8)$ & $13.2(12.0-14.4)$ & $12.5(11.0-14.0)$ & $<0.0001$ \\
\hline $\mathrm{SpO}_{2}(\%)^{*}$ & nd & $\begin{array}{c}98(97-99) \\
\quad(n=87)\end{array}$ & $\begin{array}{c}98(97-98) \\
(n=132)\end{array}$ & $\begin{array}{c}98(97-99) \\
(n=154)\end{array}$ & 0.0008 \\
\hline Body temperature (in Celsius)* & nd & $\begin{array}{c}37.2(36.8-37.5) \\
\quad(n=112)\end{array}$ & $\begin{array}{c}36.8(36.4-37.2) \\
\quad(n=158)\end{array}$ & $\begin{array}{c}36.8(36.5-37.4) \\
\quad(n=167)\end{array}$ & 0.0001 \\
\hline Disodium cromoglycate inhalation & nd & nd & $62 \%$ & $63 \%$ & 0.88 \\
\hline Leukotriene receptor antagonist & nd & nd & $90 \%$ & $91 \%$ & 0.74 \\
\hline Inhaled corticosteroid & nd & nd & $3 \%$ & $10 \%$ & 0.005 \\
\hline
\end{tabular}

nd, not done ; URI, upper respiratory infection.

*, data are shown as median (interquartile range). 


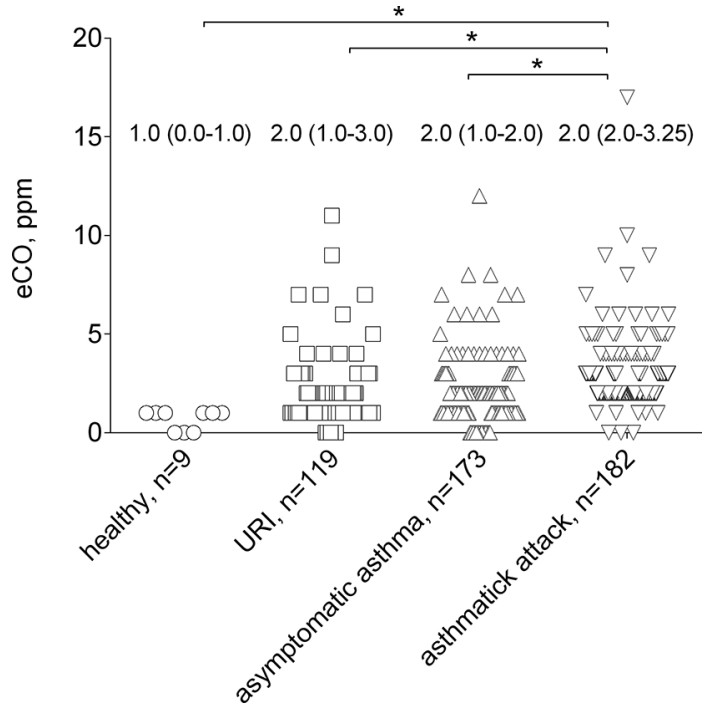

Figure 3. eCO levels of infants and toddlers

The eCO level during asthma attacks in asthmatic infants and toddlers [median (interquartile range) $=2.0(2.0-3.25), n=182]$ was significantly higher compared to that in healthy $[\mathrm{eCO}=1.0(0.0-1.0)$, $n=9$ ], URI [2.0 (1.0-3.0), $n=119$ including acute bronchitis] and asymptomatic asthma [2.0 (1.02.0), $n=173$ ]

eCO, exhaled carbon monoxide ; URI, upper respiratory infection.

$*, P<0.0001$.

(Table 2).

The eCO levels before and after the treatment of asthma

We compared the eCO levels before and after treatment in 75 infants and toddlers with asthma attack. The eCO levels during asthma attacks was elevated [3.0 (2.0-4.0) ppm] and significantly decreased after therapy $[1.0(1.0-2.0) \mathrm{ppm}, P<$ 0.0001 ; Fig. 4]. We re-examined the eCO levels 2-3 days after the prescription of anti-inflammatory drugs, not just after the inhalation therapy on the same day.

\section{The eCO levels by the activity of asthma}

In the asthmatic infants and toddlers $(n=355)$, asymptomatic asthma $(n=173)$ had eCO of $2.0\left(1.0^{-}\right.$ 2.0) ppm and asthma attack $(n=182)$ had eCO of 2.0 (2.0-3.25) ppm $(P<0.0001)$ (Fig. 5A). eCO cut-off $\geq 2 \mathrm{ppm}$ discriminated asthma attack from asymptomatic asthma with a sensitivity of $95.6 \%$, a specificity of $43.3 \%$, and an area under the curve (AUC) of 0.71 (95\% CI : 0.65-0.76, $P<0.0001$ ) (Fig. $5 B)$. In a multiple logistic regression analysis to diagnose asthma attack incorporating eCO levels, age, gender, and $\mathrm{SpO}_{2}$ values, only eCO levels independently predicted asthma attack with an odds ratio of 1.55 (95\% CI 1.29-1.86) $(P<0.001)$.

The eCO levels to diagnose asthma in infants and toddlers with respiratory symptoms

In 401 infants and toddlers reported to have respiratory symptom, those with the final diagnosis of asthma had eCO of $2.0(2.0-3.0) \mathrm{ppm}(n=285)$ and those without had eCO of $2.0(1.0-3.0) \mathrm{ppm}(n=$ 116, $P<0.0001$ ) (Fig. 6A). An eCO cut-off $\geq 3$ ppm discriminated those with a final diagnosis of asthma from those without, with a sensitivity of $38.9 \%$, a specificity of $74.1 \%$, and AUC of $0.63(95 \%$ CI : $0.56-0.69, P<0.0001$ ) (Fig. 6B).

Because children with asthma were older than those without asthma in this study (Table 1), we performed a sensitivity analysis limiting the children with respiratory symptoms aged under 2 years. The eCO of children with asthma attack [2.0 (2.03.0) ppm, $n=73$ ] was still higher than those with URI [2.0 (1.0-3.0) ppm, $n=94, P<0.0001]$. An eCO cut-off $>2$ ppm discriminated children with asthma attack from those without asthma, with a sensitivity of $93.2 \%$, a specificity of $40.4 \%$, and AUC of 0.67 (95\% CI : $0.58-0.75, P=0.0002)$. In a multiple logistic regression analysis to detect a final diagnosis of asthma incorporating eCO levels, age, gender and $\mathrm{SpO}_{2}$ values, only age independently predicted a final diagnosis of asthma with an odds ra-

Table 2. eCO levels by gender

\begin{tabular}{lrlrccc}
\hline & \multicolumn{2}{c}{ Female } & \multicolumn{2}{c}{ Male } & \multirow{2}{*}{$P$} \\
\cline { 2 - 5 } & $n$ & Median (IQR) & \multicolumn{1}{c}{$n$} & Median (IQR) & \\
\hline Healthy & 3 & $1.0(0.0-1.0)$ & 6 & $1.0(0.0-1.0)$ & 1.00 \\
URI & 43 & $2.0(1.0-3.0)$ & 76 & $2.0(1.0-3.0)$ & 0.56 \\
Asymptomatic asthma & 40 & $1.0(1.0-2.0)$ & 133 & $2.0(1.0-3.0)$ & 0.09 \\
Asthmatic attack & 61 & $2.0(3.0-3.5)$ & 121 & $2.0(2.0-3.5)$ & 0.74 \\
\hline
\end{tabular}

eCO, exhaled carbon monoxide ; IQR, interquartile range ; URI, upper respiratory infection. 


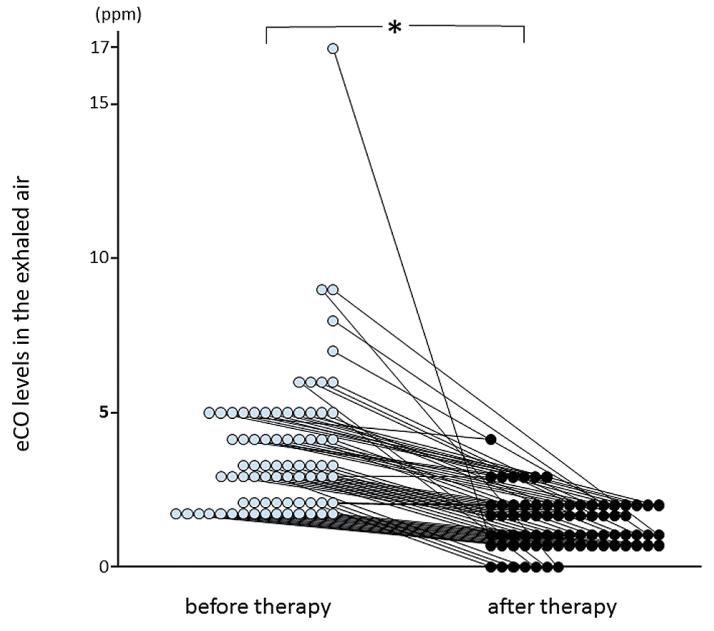

Figure 4. eCO levels in patients with asthma attacks before and after therapy $(n=75)$

$\mathrm{eCO}$ [median (interquartile range)] before therapy was $3.0(2.0-4.0) \mathrm{ppm}$ and eCO after therapy was $1.0(1.0-2.0) \mathrm{ppm}(P<0.0001)$. $\mathrm{eCO}$, exhaled carbon monoxide.

tio of 7.21 (95\% CI 4.53-11.49) $(P<0.001)$. Higher eCO levels had barely insignificant association with a final diagnosis of asthma (odds ratio and 95\% CI were 1.22 and $0.98-1.53, P=0.07$ ).

\section{Discussion}

In this prospective observational study in a primary care setting, we showed that eCO measurements were feasible in infants and toddlers, utilizing a combination of commercially available devices with some modifications.

Fractional exhaled nitric oxide concentration (FeNO) is used in asthmatic infants and toddlers universally $^{17,21-23)}$. As far as we know, however, the instrument of FeNO measurement for infants and toddlers is not readily available in Japan. We investigated measurement of eCO to reflect chronic airway inflammation of bronchial asthma using commercially available instruments ${ }^{15,16)}$. Previous reports showed that airway inflammation leads to an increased expression of HO-1 in the airway, resulting in an elevation of eCO in adult asthma patients ${ }^{4,6)}$, and school-age and preschool children ${ }^{14-16)}$ with persistent asthma. Measurements of FeNO through a mask and nonrebreathing valve in infants and toddlers was reported ${ }^{24)}$. The ATS (American Thoracic Society) in 2005 recommended off-line measurement of FeNO using one tidal breath, but it was not standardized ${ }^{25-27)}$.

At first, we tried to sample through the mask with rebreathing valve covering the nose and
A

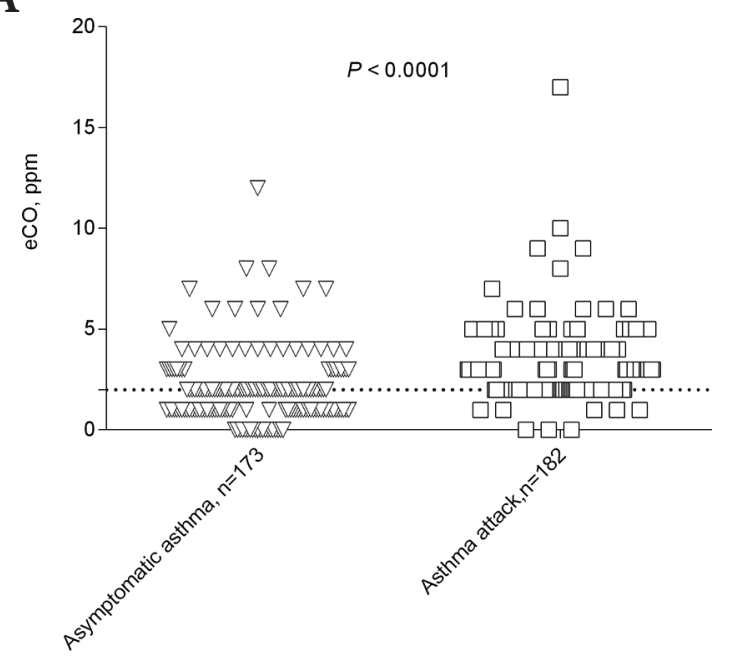

B

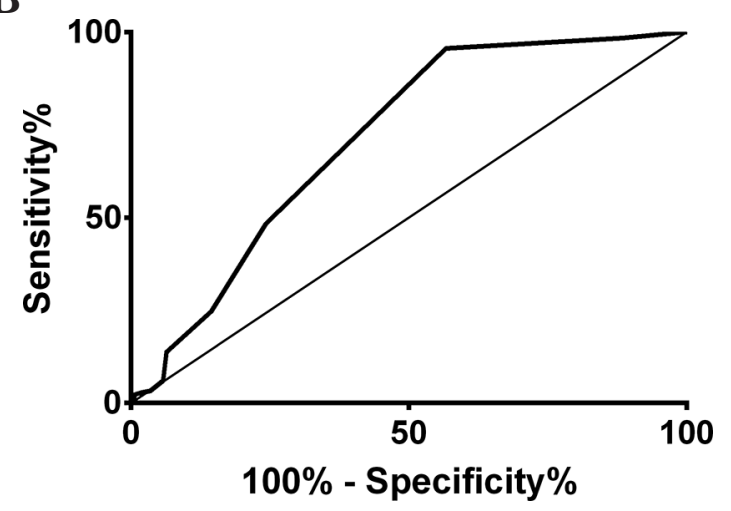

Figure 5. ROC curve of eCO to evaluate asthma activity in infants and toddlers diagnosed with asthma

A, distribution of eCO in asthmatic infants and toddlers by presence or absence of asthma attack. Dotted line indicates the cut-off $\geq 2 \mathrm{ppm}$, with a sensitivity of $95.6 \%$ and specificity of $43.3 \%$.

$\mathrm{B}$, the ROC curve to diagnose asthma attack from asymptomatic asthma in infants and toddlers diagnosed as asthma with an AUC of 0.71 (95\% CI : $0.65-0.76, P<0.0001)$.

AUC, area under the curve; CI, confidence interval ; eCO, exhaled carbon monoxide ; ROC, receiver operating characteristics.

mouth. However, we observed that the rebreathing valve interfered with breathing in infants and toddlers. Thus, we devised a per os sampling method, using a mask without a non-rebreathing valve, enabling the collection of eCO of infants. Using this new technique, we applied eCO measurements in infants and toddlers. We showed that $\mathrm{eCO}$ of infants and toddlers with asthma attacks was higher than with those having asymptomatic asthma, URI, or good health. However, there were considerable overlaps of eCO levels among children 
A

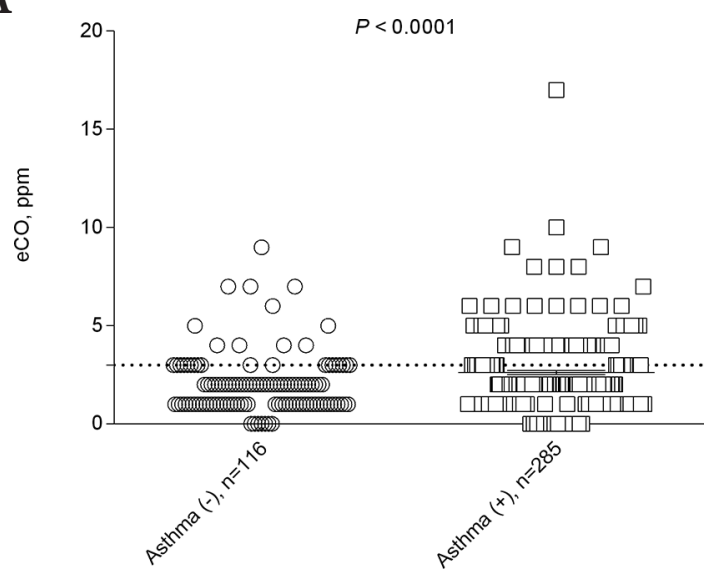

B

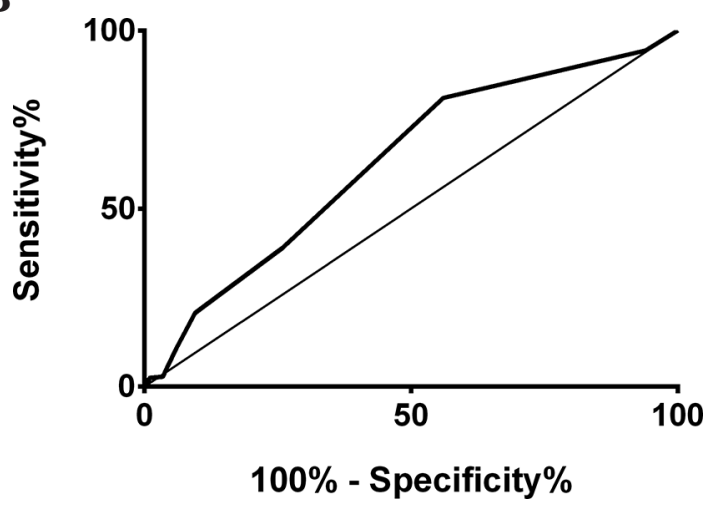

Figure 6. eCO to diagnose asthma in infants and toddlers with any respiratory symptoms

A, distribution of eCO in infants and toddlers with any respiratory symptoms by diagnosis of asthma. Dotted line indicates the cut-off $0 \geq 3 \mathrm{ppm}$, with a sensitivity of $38.9 \%$ and specificity of $74.1 \%$.

$\mathrm{B}$, the ROC curve to diagnose asthma in infants and toddlers with any respiratory symptoms with an AUC of 0.63 (95\% CI : $0.56-0.69, P<0.0001)$. AUC, area under the curve ; CI, confidence interval ; eCO, exhaled carbon monoxide ; ROC, receiver operating characteristics.

with asthma attacks, asymptomatic asthma, and URI. An eCO cut-off $\geq 2$ ppm discriminated children with asthma attack from those without attacks with a sensitivity of $95.6 \%$, a specificity of $43.3 \%$, and an area under the curve (AUC) of 0.71 (95\% CI : $0.65-0.76, P<0.0001)$. eCO levels independently predicted asthma attack in a multiple logistic regression analysis adjusted for age, gender and $\mathrm{SpO}_{2}$ values. One may reasonably consider that symptoms are controlled with eCO lower than 2 ppm in infants and toddlers known to have asthma. Among patients who presented with any respiratory symptoms, eCO cut-off $\geq 3$ ppm discriminated children with a final diagnosis of asthma from those without, with a sensitivity of $38.9 \%$, a specificity of $74.1 \%$, and AUC of 0.63 (95\% CI : $0.56-0.69$, $P<0.0001)$. The higher eCO level $(\geq 3 \mathrm{ppm})$ may warrant further investigation to diagnose asthma in infants and toddlers. However, one should take care about patients' age, considering the results of a multiple logistic regression analysis in which age was a stronger predictor of a final diagnosis of asthma than eCO levels. Abd EL Khalek et al. reported that a cut-off value of $3 \mathrm{ppm}$ for eCO was useful in distinguishing the exacerbation group from the quiescent group among children with chronic lung disease $^{28)}$. Further, several groups reported the use of a CO-Stat Meter (Natus Company, currently not available in Japan) to measure end-tidal carbon monoxide (ETCO) for the diagnosis of bronchopulmonary dysplasia in newborn infants at a cut-off value of 2 $\mathrm{ppm}^{29-31)}$. Elliott reported that single-breath FeNO (SB-FENO) concentration $\geq 30 \mathrm{ppb}$ predicted persistence of wheezing at age 3 years with a sensitivity of $77 \%$, a specificity of $94 \%$, and an area under the curve (AUC) of $0.86(95 \% \mathrm{CI}: 0.74-0.98)^{23)}$. These results and several previous reports support the clinical usefulness of eCO measurements in infants and toddlers in certain circumstances.

FeNO is widely used and considered superior to $\mathrm{eCO}$ in the diagnosis as well as management of asthma. However, there are several drawbacks in using FeNO. Currently, FeNO measurement requires forced exhalation which is impractical for infants and toddlers. The NiOX device to measure FeNO is 600,000 yen +2000 yen/test kit in Japan. $\mathrm{PiCO}+$ smokerlyzer is 118,000 yen. FeNO measurement for ${ }^{32)}$ infants and toddlers need Sievers ${ }^{\circledR}$ NOA 280 chemiluminescence analyser (GE Analytical Instruments ; Boulder, CO, USA) which is about $7,100,000$ yen in Japan and its breath sampling bag is 3,300 yen. We used a disposable breath sampling bag used for the 13-C urea exhaled test (Otsuka Pharmaceutical, Tokyo, Japan) ${ }^{15)}$ priced at 60 yen/ bag. The diagnostic ability of FeNO is superior to eCO ; however, it is too expensive and complicated to use in primary care clinics. eCO measurement has comparable diagnostic ability with better availability compared to FeNO and we believe that eCO measurement has its role in the management of asthmatic infants and toddlers in primary care practice or in low-resource settings ${ }^{33}$. However, our new method may be applicable to FeNO measurements, resulting in improved availability and lower costs.

The elevated eCO concentrations during asthma attacks were significantly decreased after thera- 
py in episodic asthma. We could not measure eCO just after inhalation combination therapy with salbutamol and SCG, but measured it at 2-3 days after the prescription of anti-inflammatory drugs. The mechanism of the decline in eCO levels after asthmatic therapy remains unclear, but it could be postulated as follows : (1) as a potent bronchodilator, a combination therapy with salbutamol and SCG increases airflow ${ }^{19)}$, resulting in decrease in eCO concentrations ; and (2) anti-inflammatory drugs following the JPGL 2012 guideline ${ }^{18)}$ step 1 may alleviate airway inflammation and decrease the production of $\mathrm{CO}$.

SCG has not only anti-inflammatory actions but also potentiating actions of the smooth muscle-relaxing properties of salbutamol in the airway ${ }^{19)}$. Silkoff et al. reported a flow dependency of NO concentrations in exhaled air ${ }^{34)}$. We previously performed expiratory peak flow measurements during mild asthma attacks in 4 of 22 patients (>8-year-old schoolchildren) and found a negative correlation between peak flow rate and eCO concentration ${ }^{16)}$. In addition to the decrease of $\mathrm{CO}$ production from the airway, the restored airflow may be one of the mechanisms underlying decrease in the eCO levels after inhalation therapy with salbutamol and SCG, although we could not obtain PEF data in infants and toddlers to support this assumption. It remains controversial, however, whether there is a simple flow dependency in the eCO concentration in patients with airway obstruction ${ }^{35,36)}$. We should be prudent in interpreting the eCO levels in cases complicated with allergic rhinitis, because the eCO levels increase in this condition ${ }^{10,37)}$.

\section{Limitations}

This study was conducted at a single community pediatric clinic. The eCO results were not blinded and might influence the diagnosis of asthma. Relatively small numbers in this study preclude detailed sub-analyses of the results.

Due to the nature of this observational study, characteristics of the groups were not well-controlled. There was no significant gender difference in the eCO levels within subgroups (Table 2). The infants and toddlers in the asthma attack group had been taking an inhaled corticosteroid more often (Table 1) ; however, those taking an inhaled corticosteroid had higher eCO levels, which makes the possibility that controller medications might influence the eCO levels unlikely. The infants and toddlers diagnosed with asthma were older than those without. However, sensitivity analysis limiting the children to those aged less than 2 years gave similar results, which support the usefulness of eCO measurements in infants and toddlers.

\section{Conclusion}

Measuring eCO is feasible in asthmatic infants and toddlers by modifying commercially available devices, and may be useful to assess the activity and treatment responsiveness in infants and toddlers with asthma, and to diagnose asthma in those with respiratory symptoms.

\section{Acknowledgements}

We thank the children and their parents, and the asthmatic outpatients attending our children's clinic, for participating in this study. We wish to thank Professor Takashi Ohrui (Division of Respiratory Medicine, Tohoku Medical and Pharmaceutical University), Professor Mutsuo Yamaya (Department of Advanced Preventive Medicine for Infectious Disease, Tohoku University Graduate School of Medicine) and Honorary Professor Hidetada Sasaki (Tohoku University Graduate School of Medicine) for helpful advice pertaining to the manuscript. We also thank Dr. Garry Heterick for his assistance in preparing the English-language manuscript. We also thank Mr. Tomohiro Ohara for assistance in preparing the illustrations. Finally, we are deeply grateful to the staff of our clinic.

\section{Disclosure}

The authors declare no conflicts of interest.

\section{Author contribution}

Y.O. designed the study and collected data ; Y.O. and T.O. analyzed data and wrote the manuscript ; K.H. and M.H. gave technical support and conceptual advice. All the authors read and approved the final manuscript.

\section{References}

1. Kharitonov SA, Yates D, Robbins RA, Logan-Sinclair R, Shinebourne EA, Barnes PJ. Increased nitric oxide in exhaled air of asthmatic patients. Lancet, 343 : 133-135, 1994.

2. Yamada N, Yamaya M, Okinaga S, et al. Protective effects of heme oxygenase-1 against oxidant-induced injury in the cultured human tracheal epithe- 
lium. Am J Respir Cell Mol Biol, 21 : 428-435, 1999.

3. Otterbein L, Sylvester SL, Choi AM. Hemoglobin provides protection against lethal endotoxemia in rats : the role of heme oxygenase- 1 . Am J Respir Cell Mol Biol, 13 : 595-601, 1995.

4. Horvath I, Donnelly LE, Kiss A, Paredi P, Kharitonov SA, Barnes PJ. Raised levels of exhaled carbon monoxide are associated with an increased expression of heme oxygenase- 1 in airway macrophages in asthma: a new marker of oxidative stress. Thorax, 53 : 668-672, 1998.

5. Jarvis MJ, Russell MA, Saloojee Y. Expired air carbon monoxide : a simple breath test of tobacco smoke intake. Br Med J, 281 : 484-485, 1980.

6. Zayasu K, Sekizawa K, Okinaga S, Yamaya M, Ohrui T, Sasaki H. Increased carbon monoxide in exhaled air of asthmatic patients. Am J Respir Crit Care Med, 156 : 1140-1143, 1997.

7. Horvath I, Loukides S, Wodehouse T, Kharitonov SA, Cole PJ, Barnes PJ. Increased levels of exhaled carbon monoxide in bronchiectasis : a new marker of oxidative stress. Thorax, 53 : 867870, 1998.

8. Yamaya M, Sekizawa K, Ishizuka S, Monma M, Mizuta K, Sasaki H. Increased carbon monoxide in exhaled air of subjects with upper respiratory tract infections. Am J Respir Crit Care Med, 158 : 311-314, 1998.

9. Maines MD. The heme oxygenase system : a regulator of second messenger gases. Annu Rev Pharmacol Toxicol, 37 : 517-554, 1997.

10. Monma M, Yamaya M, Sekizawa K, et al. Increased carbon monoxide in exhaled air of patients with seasonal allergic rhinitis. Clin Exp Allergy, 29 : 1537-1541, 1999.

11. Yasuda H, Yamaya M, Yanai M, Ohrui T, Sasaki H. Increased blood carboxyhaemoglobin concentrations in inflammatory pulmonary diseases. Thorax, 57 : 779-783, 2002.

12. Yasuda H, Sasaki T, Yamaya M, et al. Increased arteriovenous carboxyhemoglobin differences in patients with inflammatory pulmonary diseases. Chest, 125 : 2160-2168, 2004.

13. Yasuda H, Ebihara S, Yamaya M, Mashito Y, Nakamura M, Sasaki H. Increased arterial carboxyhemoglobin concentrations in elderly patients with silicosis. J Am Geriatr Soc, 52 : 1403-1404, 2004.

14. Uasuf CG, Jatakanon A, James A, Kharitonov SA, Wilson NM, Barnes PJ. Exhaled carbon monoxide in childhood asthma. J Pediatr, 135 : 569-574, 1999.

15. Ohara Y, Ohara T, Ohrui T, et al. Exhaled carbon monoxide levels in preschool-age children with episodic asthma. Pediatr Int, 54 : 227-232, 2012.
16. Ohara Y, Ohrui T, Morikawa T, et al. Exhaled carbon monoxide levels in school-age children with episodic asthma. Pediatr Pulmonol, 41: 470474, 2006.

17. Zanconato S, Scollo M, Zaramella C, Landi L, Zacchello F, Baraldi E. Exhaled carbon monoxide levels after a course of oral prednisone in children with asthma exacerbation. J Allergy Clin Immunol, 109 : 440-445, 2002.

18. Nishimuta T, Kondo N, Hamasaki Y, Morikawa A, Nishima S. Japanese guideline for childhood asthma. Allergol Int, 60 : 147-169, 2011.

19. Furusho K, Nishikawa K, Sasaki S, Akasaka T, Arita M, Edwards A. The combination of nebulized sodium cromoglycate and salbutamol in the treatment of moderate-to-severe asthma in children. Pediatr Allergy Immunol, 13 : 209-216, 2002.

20. Morikawa A, Nishima S. New Japanese pediatric guidelines for the treatment and management of bronchial asthma. Pediatr Int, 49 : 1023-1031, 2007.

21. AmericanThoracicSociety, EuropeanRespiratorySociety. ATS/ERS recommendations for standardized procedures for the online and offline measurement of exhaled lower respiratory nitric oxide and nasal nitric oxide, 2005. Am J Respir Crit Care Med, 171 : 912-930, 2005.

22. Baraldi E, de Jongste JC. Measurement of exhaled nitric oxide in children, 2001. Eur Respir J, 20 : 223-237, 2002.

23. Elliott M, Heltshe SL, Stamey DC, Cochrane ES, Redding GJ, Debley JS. Exhaled nitric oxide predicts persistence of wheezing, exacerbations, and decline in lung function in wheezy infants and toddlers. Clin Exp Allergy, 43 : 1351-1361, 2013.

24. van Mastrigt E, Gabriele C, de Jongste JC. Exhaled nitric oxide in infants - what is a nice test like FENO doing in a place like this? Semin Respir Crit Care Med, 28 : 264-271, 2007.

25. Recommendations for standardized procedures for the on-line and off-line measurement of exhaled lower respiratory nitric oxide and nasal nitric oxide in adults and children-1999. Am J Respir Crit Care Med, 160 : 2104-2117, 1999.

26. Baraldi E, Dario C, Ongaro R, et al. Exhaled nitric oxide concentrations during treatment of wheezing exacerbation in infants and young children. Am J Respir Crit Care Med, 159 : 1284-1288, 1999.

27. Avital A, Uwyyed K, Berkman N, Godfrey S, BarYishay E, Springer C. Exhaled nitric oxide and asthma in young children. Pediatr Pulmonol, 32: 308-313, 2001.

28. Abd El Khalek KA, El Seify MY, Youssef OI, Badr MM. Diagnostic value of exhaled carbon monoxide as an early marker of exacerbation in children with chronic lung diseases. ISRN Pediatr, 2012 : 
859873, 2012.

29. Krediet TG, Cirkel GA, Vreman HJ, et al. Endtidal carbon monoxide measurements in infant respiratory distress syndrome. Acta Paediatr, 95 : 1075-1082, 2006.

30. May C, Patel S, Peacock J, Milner A, Rafferty GF, Greenough A. End-tidal carbon monoxide levels in prematurely born infants developing bronchopulmonary dysplasia. Pediatr Res, 61 : 474-478, 2007.

31. Blok CA, Krediet TG, Kavelaars A, Koopman-Esseboom C, Vreman HJ, Van Bel F. Early end-tidal carbon monoxide levels and neurodevelopmental outcome at 3 years 6 months of age in preterm infants. Developmental Medicine \& Child Neurology, 53 : 1113-1118, 2011.

32. Collins SA, Pike KC, Inskip HM, et al. Validation of novel wheeze phenotypes using longitudinal airway function and atopic sensitization data in the first 6 years of life : evidence from the Southampton Women's survey. Pediatr Pulmonol, 48 :
683-692, 2013.

33. Pereira AA, Pollard SL, Locke R, et al. Association between exhaled carbon monoxide and asthma outcomes in Peruvian children. Respir Med, 145 : 212-216, 2018.

34. Silkoff PE, McClean PA, Slutsky AS, et al. Marked flow-dependence of exhaled nitric oxide using a new technique to exclude nasal nitric oxide. Am J Respir Crit Care Med, 155 : 260-267, 1997.

35. Zetterquist W, Marteus H, Johannesson M, et al. Exhaled carbon monoxide is not elevated in patients with asthma or cystic fibrosis. Eur Respir J, 20 : 92-99, 2002.

36. Beck-Ripp J, Latzin P, Griese M. Exhaled carbon monoxide is not flow dependent in children with cystic fibrosis and asthma. Eur J Med Res, 9 : 518-522, 2004.

37. Jesenak M, Banovcin P, Havlicekova Z, Dobrota D, Babusikova E. Factors influencing the levels of exhaled carbon monoxide in asthmatic children. J Asthma : 1-7, 2014. 